TITLE:

\title{
ACTIVITY RHYTHM OF THE SEA- PEN, CAVERNULARIA OBESA VALENCIENNES, UNDER TEMPERATURE AND LIGHT CYCLES
}

\author{
$\operatorname{AUTHOR}(\mathrm{S})$ : \\ Imafuku, Michio
}

\section{CITATION:}

Imafuku, Michio. ACTIVITY RHYTHM OF THE SEA-PEN, CAVERNULARIA OBESA

VALENCIENNES, UNDER TEMPERATURE AND LIGHT CYCLES. PUBLICATIONS OF THE SETO MARINE BIOLOGICAL LABORATORY 1980, 25(1-4): 119-130

ISSUE DATE:

1980-02-29

URL:

http://hdl.handle.net/2433/175989

RIGHT: 


\title{
AGTIVITY RHYTHM OF THE SEA-PEN, GAVERNULARIA OBESA VALENGIENNES, UNDER TEMPERATURE AND LIGHT GYCLES
}

\author{
MichIo IMAFUKU1) \\ Department of Zoology, Faculty of Science, Kyoto University
}

With Text-figures $1-3$

Circadian rhythms are generally known to be entrained to light cycles, and in some poikilothermic animals living in terrestrial region also to temperature cycles. The entraining effect of temperature cycles may be attributable to the fact that the daily fluctuation of temperature on the land is rather clear and regular closely related to the natural light change. On the other hand, the daily fluctuation of temperature in the sea is not so clear as in the terrestrial region, being generally very small or perfectly null depending on the depth. So, it is interesting to examine the effect of temperature cycle on the circadian rhythm of marine organisms, to make it clear whether the effect of temperature cycle is dependent on species, or generally seen on the circadian rhythms of different species of poikilothermic animals.

The author made an experiment concerning this problem in a series of experiments with using of the sea-pen, Cavernularia obesa, a marine colonial coelenterate living on the sand bottom of shallow water, and obtained data somewhat unclear with difference among colonies in behavior under the temperature cycle (Imafuku, 1973). Thus, he repeated the experiment on the same species, recording its behavior continuously for about four months through under temperature cycles of various levels of temperature, and also examining the effect of light cycle.

\section{Material and Method}

A colony of the sea-pen, Cavernularia obesa Valenciennes, collected from the sand bottom at $2 \mathrm{~m}$ deep near Hatakejima Island in Tanabe Bay in December 1973 was used. The body weight in the contracted state was $43 \mathrm{~g}$ at the time of collecting.

The animal had been kept in the laboratory aquarium for 2 months before the beginning of the experiments opened on February 7 and closed on June 10. During the experiments it was kept in the glass vessel $(12 \mathrm{~cm}$ high, $8 \mathrm{~cm}$ in diameter) filled with sand, and immersed in the circulating sea water in a glass cylinder $(50 \mathrm{~cm}$ high, 10.3 $\mathrm{cm}$ in diameter) as shown in Fig. 1 .

1) Contributions from the Seto Marine Biological Laboratory, No. 654.

Publ. Seto Mar. Biol. Lab., XXV (1/4), 119-130, 1980. ～(Article 9) 




Fig. 1. Method for recording the expansion-contraction behavior of the sea-pen placed in the circulating sea water.

The animal was continuously exposed to temperature cycles under the condition of continuous dim light or light cycle. The temperature cycle was made by controlling the temperature of the circulating sea water; heating the water only for 12 hours in a day and uncontrolling it for the other 12 hours, or heating it at two different temperatures each lasting 12 hours. The temperature of the water was affected to some extent by the ambient atmospheric temperature, especially when it was uncontrolled. Each temperature measured in each phase of the temperature cycle is shown in Fig. 2. The level of the temperature cycle was raised as day elapsed. The phase of the temperature cycle was either "daytime-warm", the high temperature phase from 6:00 to 18:00 and the low temperature phase from 18:00 to 6:00, or "nighttime-warm", the high temperature phase from 18:00 to $6: 00$ and the low temperature phase from 6:00 to 18:00.

The light condition was continuous dim light or in some experiment light cycle. The light cycle was made by turning on and off a 40-W daylight fluorescent lamp which produced a light intensity of 1000 lux at the top of the cylinder and of 300 lux at the level of the sand surface aside the cylinder. A small pilot lamp was continuously on throughout the experiments as a back ground illumination of ca. 1 lux which enabled the experimenter to work in the "dark" period. Thus, the light condition was either the continuous dim light by the pilot lamp or the alternation of 12 hours light by the

Fig. 2. Records of the behavior of the sea-pen provided with the temperature cycle in the continuous dim light or the light cycle. The active expanded state is expressed with a trough and the inactive contracted state with a horizontal straight line. Light condition is shown with a bar above the graph; the light phase with white and the dim light phase with black. Temperature condition is shown at the bottom with numbers indicating each temperature. Arabic numerals at the top are date and Roman numerals are numbers of Experiments. 




Fig. $2(1)$
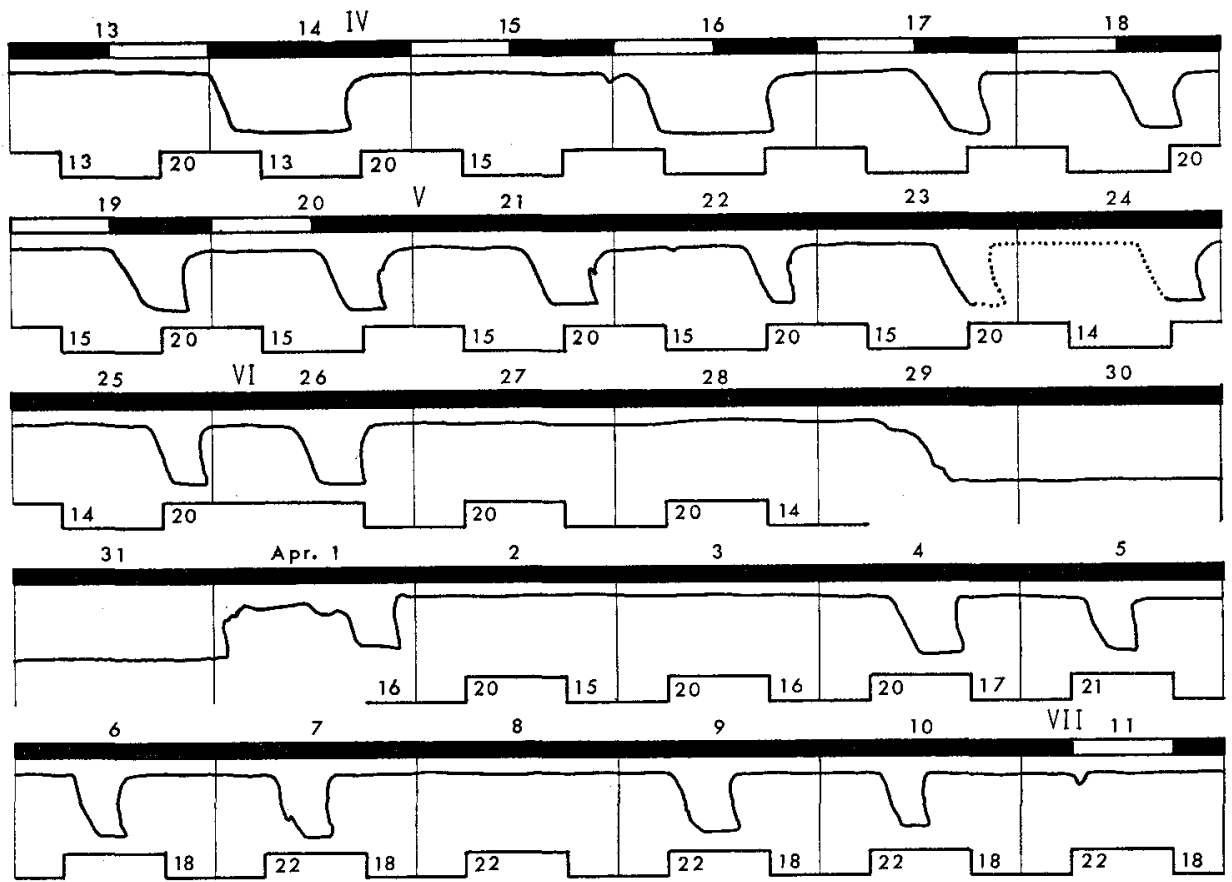

Fig. $2(2)$ 

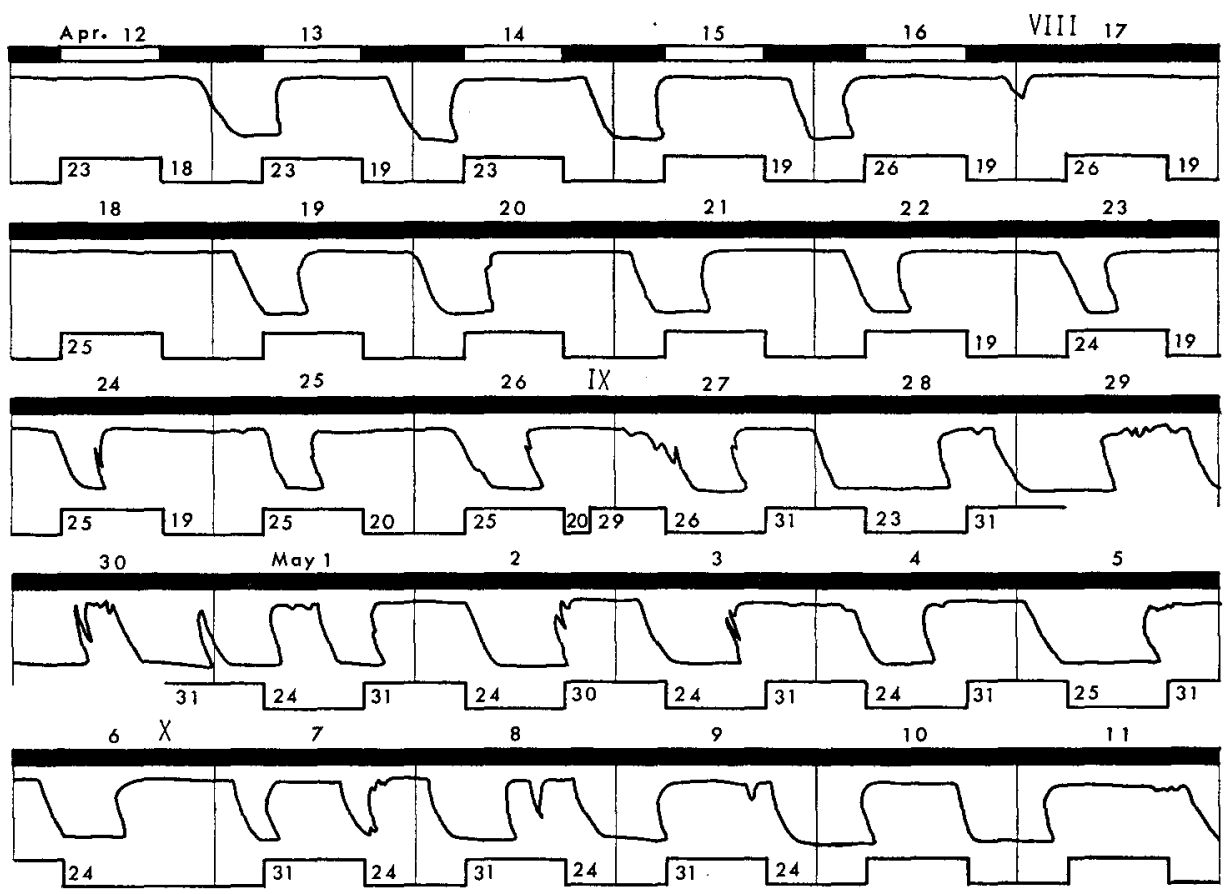

Fig. 2 (3)


Fig. 2(4) 
fluorescent lamp and 12 hours dim light by the pilot lamp.

The method for recording the expansion-contraction behavior of the sea-pen was described in detail previously and is briefly illustrated in Fig. 1.

\section{Results}

The behavior of the sea-pen was recorded throughout the experiments and is shwon in Fig. 2 after February 11. To make the behavior in each experiment comprehensive, schematic figures are given in Fig. 3.

Experiment I (February 7-14)

Firstly the effect of temperature cycle on the activity rhythm of the sea-pen was examined in the continuous dim light. The sea-pen was subjected to the temperature cycle composed of a high temperature phase at $18-19^{\circ} \mathrm{C}$ and a low temperature phase at $12-13^{\circ} \mathrm{C}$ after February 7. The high temperature phase was adjusted to the daytime.

Under this condition, the sea-pen showed the active expanded state in the high temperature phase and the inactive contracted state in the low temperature phase.

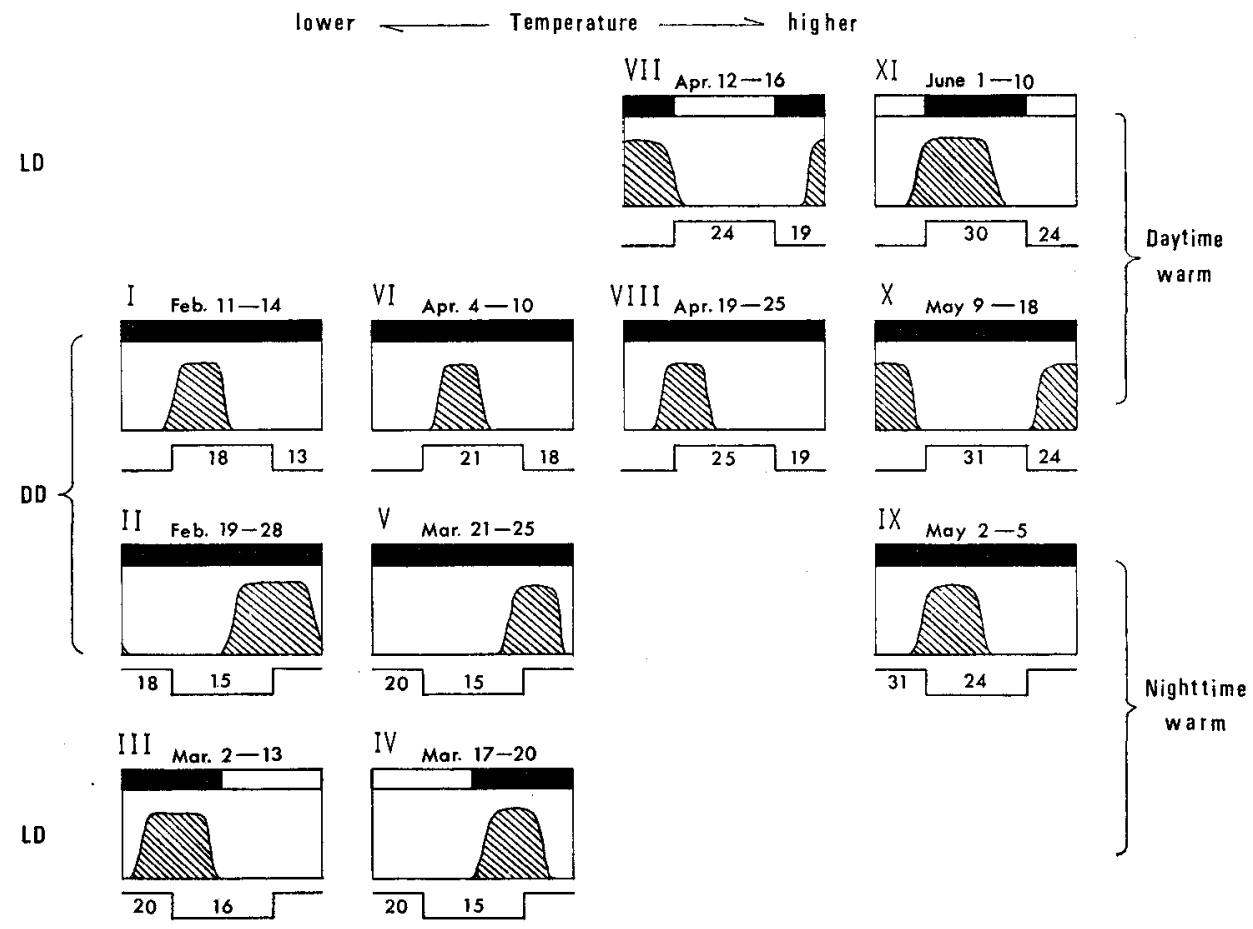

Fig. 3. Schematic illustration of the behavior in each experiment. The average active state shown with oblique lines is made from the original records based on the mean time of the beginning and the end of the activity in the term indicated at the top of the graph. Indications of the light and temperature conditions are same as in Fig. 2. 
Experiment II (February 14-28)

To examine whether the temperature cycle truely has an entraining effect on the activity rhythm of this animal or not, its behavior was observed in the temperature cycle reversed from the previous phase. The phase reversion was made on February 14 by lengthening the high temperature phase. So, the sea-pen was exposed to the temperature cycle composed of a high temperature phase at $17-18^{\circ} \mathrm{C}$ in the nighttime and a low temperature phase at $13-16^{\circ} \mathrm{C}$ in the daytime under the condition of $\operatorname{dim}$ light.

Under the reversed temperature cycle it sometimes maintained the contracted state for one to two days but tended to show the active expanded state around the beginning of the high temperature phase. Thus, the activity rhythm of the sea-pen was almost reversed by reversing the ambient tepmerature cycle. That is, it was suggested that the temperature cycle truely entrains the activity rhythm of the sea-pen.

Experiment III (March 1-13)

In this experiment, it was examined which of the two cycles, temperature cycle or light cycle, was more effective on the activity rhythm of this nocturnal animal. As the active expanded state was found to appear around the beginning of the high temperature phase in Experiment II, the light phase of the light cycle was settled on this time from 12:00 to 24:00 in this experiment. The temperature cycle was continued from the previous experiment, with the high temperature phase at $18-21^{\circ} \mathrm{C}$ in the nighttime and the low temperature phase at $13-17^{\circ} \mathrm{G}$ in the daytime.

In the simultaneous light and temperature cycles, the sea-pen showed the active expanded state in the dark phase of the light cycle, but neither in the high temperature phase as seen in Experiment I nor around the beginning of the high temperature phase as observed in Experiment II. That is, the sea-pen followed exclusively the light cycle neglecting the temperature cycle in the simultaneous light and temperature cycles.

Experiment VI (March 14-20)

The phase of the light cycle was reversed by lengthening the dark phase on March 14. The temperature cycle was continued in the same phase as in the previous experiment, with the high temperature phase at $20^{\circ} \mathrm{C}$ in the nighttime and the low temperature phase at $15^{\circ} \mathrm{C}$ in the daytime.

In the first three days of this condition, the sea-pen showed somewhat prolonged rhythm but it soon recovered the normal rhythm, adjusting the active expanded state to the dark phase of the light cycle after March 17. The result of this experiment as well as that of Experiment III revealed that the light cycle is more effective on the activity rhythm of the sea-pen than the temperature cycle.

Experiment V (March 21-25)

The behavior of the sea-pen was observed under the temperature cycle in the dim light again. The temperature cycle was the alternation of a high temperature phase at $20^{\circ} \mathrm{C}$ in the nighttime and a low temperature phase at $14-15^{\circ} \mathrm{C}$ in the daytime.

In this condition the sea-pen showed a regular rhythm synchronized with the 
temperature cycle adjusting the active expanded state to the first half of the high temperature phase.

Experiment VI (March 26-April 10)

The phase of the temperature cycle was reversed on March 26. Thus, the seapen was exposed to the high temperature phase at $20-22^{\circ} \mathrm{C}$ in the daytime and the low temperature phase at $14-18^{\circ} \mathrm{C}$ in the nighttime. The continuous dim light was followed from the previous experiment. The water supply was stopped by accident on March 29 and recovered April 1. During this period, the sea-pen was comprised in the standing water, the temperature of which was thought to be almost same as that of the surrounding air at about $15^{\circ} \mathrm{C}$.

On March 26, the day of phase reversion of the temperature cycle, the sea-pen expanded once in the high temperature phase, but it maintained the contracted state through the following two days. During the period when the water supply was stopped, the sea-pen tended to be in the expanded state without showing the expansion-contraction rhythm. After the fresh water was introduced into the cylinder again, it became contracted for two days and then it recovered its normal expansion-contraction rhythm on April 4, showing the active expanded state in the high temperature phase.

\section{Experiment VII (April 11-16)}

The behavior of the sea-pen was observed again in the simultaneous light and temperature cycles after April 11. The light phase of the light cycle was settled on the high temperature phase from 6:00 to 18:00 in which the sea-pen was found to expand in the continuous dim light in Experiment VI. The high temperature was at $22-26^{\circ} \mathrm{C}$ and the low temperature at $18-19^{\circ} \mathrm{C}$ in this experiment.

When the sea-pen was subjected to this condition, it synchronized the active expanded state with the dark phase of the light cycle, but not with the high temperature phase as seen in Experiment VI. That is, the sea-pen followed exclusively the light cycle, the result same as in Experiments III and IV.

\section{Experiment VIII (April 17-26)}

The sea-pen was returned to the continuous dim light again under the temperature cycle continued from the previous experiment. The temperature was at $24-26^{\circ} \mathrm{C}$ in the high temperature phase and at $19-20^{\circ} \mathrm{C}$ in the low temperature phase.

The active expanded state of the sea-pen shifted to the daytime when the high temperature appeared in the continuous dim light. So that, the sea-pen was warmactive in this experiment as in Experiments I, II, V and VI.

\section{Experiment IX (April 27-May 5)}

In this experiment the behavior of the sea-pen was observed in the temperature cycle of relatively high temperature level, the high temperature phase at $29-31^{\circ} \mathrm{C}$ and the low temperature phase at $23-26^{\circ} \mathrm{C}$. From April 29 to 30 the water supply was stopped.

When the sea-pen was exposed to the temperature cycle of high temperature level, it expanded in the low temperature phase. Thus, the result was utterly opposite to 
those of Experiments I, II, V, VI and VIII in which it expanded around the beginning of or in the high temperature phase.

Experiment X (May 6-17)

The phase of the temperature cycle was reversed in the continuous dim light condition on May 6. The high temperature phase was at $31-32^{\circ} \mathrm{C}$ and the low temperature phase was at $24-25^{\circ} \mathrm{C}$ in this experiment.

The first two to three days of the reversed temperature cycle, the sea-pen showed somewhat irregular behavior but soon recovered its normal state, expanding in the low temperature phase. Therefore, it could be proved in this experiment as well as the previous one that the sea-pen is cold-active in the temperature cycle of high temperature level.

Experiment XI (May 18-June 10)

The sea-pen was exposed to the light cycle again under the temperature cycle of high temperature level. The light phase was settled on the low temperature phase in which the sea-pen was found to be active in Experiments IX and X. The high temperature phase was $29-32^{\circ} \mathrm{C}$ and the low temperature phase was $24-25^{\circ} \mathrm{C}$.

In the first seven to eight days of the new light and temperature cycles, the sea-pen exhibited somewhat irregular behavior, expanding sometimes in the light-cold phase sometimes in the dark-warm phase, but after May 27, the main activity tended to occur in the dark-warm phase. Therefore, it was found also in this experiment that the seapen followed the light cycle but not the temperature cycle, as seen in Experiments III, IV and VII.

\section{Discussion}

From the present experiments, the following could be concluded. (1) The expansion-contraction behavior of the sea-pen, Cavernularia obesa, can be synchronized with the temperature cycles, (2) when the synchronization between the temperature cycle and the activity rhythm establishes, the active expanded state comes to the high temperature phase in the temperature range below $25^{\circ} \mathrm{C}$, and to the low temperature phase in the temperature range above $25^{\circ} \mathrm{C}$, and (3) the sea-pen follows exclusively the light cycle neglecting the temperature cycle when it is exposed to the simultaneous light and temperature cycles.

\section{Entrainment to Temperature Cycle}

As to the first conclusion, it may disagree with the result of the experiment reported previously in 1973. In the previous experiment, the behavior of four colonies of the same species were observed under the temperature cycle of $26^{\circ} \mathrm{C}$ and $30^{\circ} \mathrm{C}$ for 15 days, and found to be somewhat irregular with difference among colonies. Two colonies out of the four, however, tended to expand in the low temperature phase of the temperature cycle at the end of the recording. As long as the behavior of these two colonies is concerned, the result of the previous experiment agrees with that of the present experiment because the sea-pen expanded in the low temperature phase under the 
temperature cycle of high temperature range. As to the behavior of the other two colonies, it may be explained with difference among the colonies in response to temperature cycle. Similar individual difference to the temperature cycle has been observed in the activity rhythm of lizards and mice, as summerized by Hoffman (1969). Besides, different colonies of the sea-pen did not always exhibit a uniform behavior under certain light conditions (Mori, 1960). When these facts are taken into consideration, the result of the previous experiment will not always be incompatible with that of the present experiments.

In the present experiment, the activity is observed in synchrony with the temperature cycle. With respect to this phenomenon, the following two explanations may be proposed: one is that the rhythmic behavior observed under the temperature cycle is due to a simple response to a periodic stimulus such as temperature drop or rise and that the basic rhythm (circadian rhythm) is not affected by the temperature cycle, and the other is that a true entrainment of the circadian rhythm to the temperature cycle is established. The basic difference between the two is whether the circadian rhythm is truely synchronized with the temperature cycle or not. As long as the observation is made under a temperature cycle, it is unknown which of the two explanations is valid for the activity rhythm of the sea-pen. It may become clear, however, when the phase of the temperature cycle is suddenly changed; that is, if the rhythmic behavior is due to a simple response to the temperature cycle, the active state will be shifted simultaneously with the phase shift of the temperature cycle, and if the circadian rhythm is truely entrained to the temperature cycle, the original rhythm will persist more or less under the shifted temperature cycle before a perfect synchronization is attained. The latter explanation seems to be true for the activity rhythm of the sea-pen because the activity rhythm persisted for one to two days just after the phase reversion of the temperature cycle, as seen on February 15 and March 26. Therefore, the expansioncontraction behavior observed under the temperature cycle is thought to be based on the entrainment of the circadian rhythm of the sea-pen to temperature cycles.

The entrainment of the circadian rhythm to the temperature cycles is known in various species of poikilothermic animals and plants as reviewed by Bruce (1960), Sweeney and Hastings (1960), Bünning (1973) and reported by Karakashian (1968). On the other hand, it is not established in some species of homoiothermic animals such as flying squirrels, mice, hamsters and birds as summerized by Hoffmann (1969). Therefore, it is not unnatural that the circadian rhythm of the sea-pen belonging to poikilothermic animals can be entrained to temperature cycles.

The effect of temperature cycles has been examined also in the other marine animals, though not on circadian rhythms but on tidal rhythms. Alternation of different two temperatures, each lasting 6 to 6.2 hours, entrained the tidal activity rhythm of the gree crabs (Williams and Naylor, 1969) but had no effect on the activity rhythm of the littoral fish, Blennius (Gibson, 1970).

Phase of Rhythm under Temperature Cycles

When circadian rhythms are synchronized with temperature cycle, it is said that 
the state of organisms seen at night is exhibited in the low temperature phase of the temperature cycle and vice versa. Some of such examples are gathered and described by Bünning (1973) and others are seen in the cell division rhythm of Paramecium bursaria (Volm, 1964), the mating rhythm of $P$. aurelia (Karakashian, 1967) and the activity rhythm of pill bugs, Tylos granulatus (Imafuku, 1976). The similar phase relationship between the temperature cycle and the state of organisms is seen also in the activity rhythm of rats (Calhoun, 1945; Hoffmann, 1969), though the rhythm could not be truely entrained; that is, the activity itself was suppressed by the high temperature and induced by the low temperature but the endogenous rhythm persisted without being affected by the temperature cycle, which was revealed in the constant condition following the temperature cycle.

On the other hand, the situation is somewhat different in the case of the activity rhythm of the sea-pen. The phase relationship between the temperature cycle and the activity rhythm mentioned above can be held only in the temperature range above $25^{\circ} \mathrm{C}$ but not in the range below $25^{\circ} \mathrm{C}$, because this nocturnal animal became active in the high temperature phase in the temperature range below $25^{\circ} \mathrm{C}$ as seen in Experiments I, II, V, VI and VIII. This exceptional behavior of the sea-pen may be attributable to the environmental conditions of its habitat and the distribution. The sea-pen lives on the sea bottom from $20 \mathrm{~m}$ deep to the low tide line where the daily fluctuation of the temperature is thought to be rather small, probably less that $1^{\circ}$ or $2^{\circ} \mathrm{C}$. The temperature difference between day and night in the sea is said to be at most $1-2^{\circ} \mathrm{C}$ only in the surface water. When the temperature measurements of the coastal water regularly made twice a day at 9:00 a.m. and at the time of the low or high tide by the staff of the Seto Marine Biological Laboratory were referred to, they showed that the temperature difference between two measurements in a day was $0.63^{\circ} \mathrm{C}$ in average, 2.17 at maximum in February and $0.56^{\circ} \mathrm{C}$ in average, $1.63^{\circ} \mathrm{C}$ at maximum in August 1978 , though they did not always reveal the temperature difference between day and night. And, it was also found by the measurements that the morning temperature was not always lower than the evening one, suggesting the temperature of the coastal water is also affected by the other factors such as tidal current. Thus, the daily fluctuation of the temperature in the habitat of the sea-pen is thought to be not so clear as in the terrestrial region, and this seems to be the reason why the phase relationship between temperature cycle and activity rhythm maintained in terrestrial organisms can not be applied to the activity rhythm of the sea-pen.

The next question to be answered is why the sea-pen is warm active in the temperature cycle of the low temperature range and cold active in the temperature cycle of the high temperature range. Generally organisms must have a property to avoid unfavorable conditions and, as a result, it becomes active in the suitable condition. The sea-pen which is known to be distributed from Ishikari Bay at $43^{\circ} \mathrm{N}$.L, northern limit, to subtropical regions of the Pacific Ocean is expected to favor relatively warm water, and if the favorable temperature for the sea-pen coincides with about $25^{\circ} \mathrm{C}$, the behavior of this animal under the temperature cycle will be explained as follows. That is, it becomes active only around $25^{\circ} \mathrm{C}$ and hides itself in the sand bottom when the ambient temperature is lower or higher than this value. 
The Simultaneous Light and Temperature cycles

Out of many environmental factors which fluctuate diurnally in nature, the light and temperature are well known as effective zeitgebers (synchronizers). And, the latter is said to be less effective than the former (Bünning, 1973; Harker, 1964). In fact, this was applicable to the circadian rhythm of the sea-pen because the sea-pen always followed the light cycle independently of the temperature range of the temperature cycle as seen in the present Experiments III, IV, VII and XI. A result partly supporting this has been obtained on the activity rhythm of the pill bugs, Tylos (Imafuku, 1976). When this noctural isopod was subjected to the simultaneous light and temperature cycles in which the light phase was combined with the low temperature phase, it actively crawled in the dark-warm phase if the temperature cycle was alternation of $20^{\circ}$ and $30^{\circ} \mathrm{C}$ or of $25^{\circ}$ and $30^{\circ} \mathrm{C}$, but it tended to be active in the light-cold phase if the temperature cycle was alternation of $20^{\circ}$ and $25^{\circ} \mathrm{C}$. It is, therefore, said from the behavior of the sea-pen and pill bugs that the light cycle is, as a whole, a predominating zeitgeber and that the relative effectiveness of each zeitgeber depends on the magnitude of the zeitgeber, such as the amplitide (difference in light intensity or temperature) and the level (absolute light intensity or temperature) of the zeitgeber, as suggested by Hoffmann (1969) and Bünning (1973). And, the effectiveness of a zeitgeber will be dependent also on the sensitivity of animals to the zeitgeber (Imafuku, 1975). The sea-pen seems to be relatively insensitive to the temperature cycles but rather affectible to the light cycle, which may be expected from the environmental conditions of its habitat. This may be a reason why the expansion-contraction rhythm of the sea-pen is entrained exclusively to the light cycle when it was exposed to the simultaneous light and temperature cycles and can be synchronized with the temperature cycle when it was subjected only to the temperature cycle in the continuous dim light.

As to the effect of the simultaneous light and temperature cycles, elaborated experiments have been made on the phototactic rhythm of Euglena (Bruce, 1960), the eclosion rhythm of Drosophila (Pittendrigh, 1958) and the activity rhythm of the cockroach (Roberts, 1960), and it has been found from the experiments that the phase of the circadian rhythm is dependent also on the phase relationship between the light cycle and the temperature cycle employed. With respect to the activity rhythm of the sea-pen, further experiments in which attention is payed especially to such phenomenon should be needed before discussion in such detail is made.

\section{Acknowledgments}

The present author wishes to express his sincere gratitude to the staff of the Seto Marine Biological Laboratory for affording the author every facility for the works at the laboratory, especially to Mr. Hidetomo Tanase for his kindness in collecting material.

\section{Summary}

The behavior of the sea-pen, Cavernularia obesa Valenciennes was observed under the condition of temperature cycle in the dim light or the simultaneous light and temper- 
ature cycles to examine whether the circadian rhythm of the animal is entrained to these cycles or not, and the following results were obtained.

(1) The expansion-contraction behavior of the sea-pen could be synchronized with the temperature cycles. (2) When synchronization between the temperature cycle and the activity rhythm was established, the active expanded state came to the high temperature phase in the temperature range below $25^{\circ} \mathrm{C}$, and to the low temperature phase in the range above $25^{\circ} \mathrm{C}$. (3) The sea-pen followed exclusively the light cycle neglecting the temperature cycle when it was exposed to the simultaneous light and temperature cycles.

Some discussions are made of these results, especially connecting specific features in the behavior of this animal with the environmental conditions of its natural habitat.

\section{REFERENCES}

Bruce, V.G. 1960. Environmental entrainment of circadian rhythms. Cold Spring Harbor Symp. Quant Biol. 25: 29-48.

Bünning, E. 1973. “The Physiological Clock”. Rev. 3rd Ed. The English University Press, London.

Calhoun, J.B. 1945. Diel activity rhythms of the rodents, Microtus ochrogaster and Sigmodon hispidus. Ecology, 26: 251-273.

Gibson, R.N. 1971. Factors affecting the rhythmic activity of Blennius pholis L. (Teleostei). Anim. Behav., 19: $336-343$.

Harker, J.E. 1964. "The Physiology of Diurnal Rhythms". Cambridge University Press, London and New York.

Hoffmann, K. 1969. Die relative Wirksamkeit von Zeitgebern. Oecologia, 3: 184-206.

Imafuku, M. 1973. On some physiological aspects in the daily rhythmic activity of the sea-pen, Cavernularia obesa Valenciennes. Publ. Seto Mar. Biol. Lab., 20(Proc. Second Internat. Symp. Cnidaria), 431-454.

1975. Adaptation of the circadian rhythm of mating-reactivity to abnormal light-dark cycles in Paramecium bursaria. J. interdiscipl. Cycle Res., 6: 141-151.

1976. On the nocturnal behavior of Tylos granulatus Miers (Crustacea Isopoda). Publ. Seto Mar. Biol. Lab., 23: 299-340.

Karakashian, M.W. 1968. The rhythm of mating in Paramecium aurelia, syngen 3. J. cell. Physiol., 71 : 197-209.

Mori, S. 1960. Influence of environmental and physiological factors on the daily rhythmic activity of a sea-pen. Cold Spring Harbor Symp. Quant. Biol., 25: 334-344.

Pittendrigh, G.S. 1958. Perspectives in the study of biological clocks. pp. 239-268. in "Perspectives in Marine Biology". ed. A.A. Buzzati-Travesso. University of California Press, Berkeley.

Roberts, S.K. 1960. Circadian activity rhythms in cockroaches. I. The free-running rhythm in steady state. J. Cell. and Comp. Physiol., 55: 99-110.

Sweeney, B.M. and J.W. Hastings 1960. Effects of temperature upon diurnal rhythms. Cold Spring Harbor Symp. Quant. Biol., 25: 87-104.

Volm, M. 1964. Die Tagesperiodik der Zellteillung von Paramecium bursaria. Z. vergl. Physiol., 48: $157-$ 180 .

Williams, B.G. and E. Naylor 1969. Synchronization of the locomotor tidal rhythm of Carcinus. J. exp. Biol., 51 : 715-725. 